\title{
Antimicrobial-resistant Bacteria Arising from the Use of Colistin Sulfate in the Livestock (2nd edition) (Antimicrobial-resistant Bacteria)
}

\author{
Summary \\ Food Safety Commission of Japan
}

\begin{abstract}
The Food Safety Commission of Japan (FSCJ) updated a risk assessment on antimicrobial-resistant bacteria arising from the use of a veterinary medicinal product, colistin sulfate, in cattle and pigs, according to the "Assessment Guideline for the Effect of Food on Human Health Regarding Antimicrobial-Resistant Bacteria Selected by Antimicrobial Use in Foodproducing Animals" (FSCJ, September 30, 2004). Both Escherichia coli (E. coli) and Salmonella enterica subsp. enterica (Salmonella) were potential antimicrobial-resistant bacteria. In cases of occurrences of human infectious diseases due to the bacteria in foods derived from livestock, these resistant bacteria could be responsible for reduction or loss of the antibiotic treatment efficacy. FSCJ thus conducted a risk assessment of E. coli and Salmonella as identified hazards. FSCJ judged to be low on the occurrence probability and extent of selection of drug-resistant E. coli and Salmonella, due to the use of colistin sulfate in cattle and pigs, unless otherwise the use of colistin increases. The chance and extent of human exposure to the resistant bacteria were evaluated low via livestock products including pigs and cattle, as long as proper cooking practice is implemented. The degree of possible reduction or loss of clinical effectiveness against E. coli and Salmonella was evaluated as moderate. The overall estimation of the risk regarding reduction or loss of clinical effectiveness of antimicrobials in humans was low. It is necessary to keep up with the latest scientific findings and information.
\end{abstract}

\section{Conclusion in Brief}

The Food Safety Commission of Japan (FSCJ) updated a risk assessment on antimicrobial-resistant bacteria arising from the use of a veterinary medicinal product, colistin sulfate, in cattle and pigs, according to the "Assessment Guideline for the Effect of Food on Human Health Regarding Antimicrobial-Resistant Bacteria Selected by Antimicrobial Use in Food-producing Animals" (FSCJ, September 30, 2004).

In the first assessment (2017), Escherichia coli (E. coli) was identified as a hazard ${ }^{1)}$. Based on the assessment, the Ministry of Agriculture, Forestry and Fisheries (MAFF) withdrew the designation of colistin as a feed additive, and placed restriction on its use for veterinary therapeutics in 2018.

In addition to $E$. coli. in the 1st edition, Salmonella enterica subsp. enterica (Salmonella) is newly added as a hazard in the 2nd edition. In fact, monitoring on E. coli and Salmonella derived from livestock, conducted from 2000 through 2017, has suggested the retention of colistin susceptibility in almost all these bacteria. Some strains were found to contain

Published online: 30 March 2021

This is an English translation of excerpts from the original full report (February-FS/41/2021). Only original Japanese texts have legal effect. The original full report is available in Japanese at http://www.fsc.go.jp/fsciis/attachedFile/download?retrievalId=kya20200204000\&fileId=201 Abbreviation : CRE: carbapenem-resistant Enterobacteriaceae, E. coli: Escherichia coli, FSCJ: Food Safety Commission of Japan, Salmonella: Salmonella enterica subsp. enterica, MAFF: Ministry of Agriculture, Forestry and Fisheries, MDR: multidrug-resistant, MDRP: MDR Pseudomonas aeruginosa.

Suggested citation: Food Safety Commission of JAPAN. Antimicrobial-resistant Bacteria Arising from the Use of Colistin Sulfate in the Livestock (2nd edition) (Antimicrobial-resistant Bacteria). Food Safety. 2021; 9 (1) 22-24. doi: 10.14252/foodsafetyfscj.D-21-00003 
$m c r-1, m c r-3$, and/or $m c r-5$ genes in these samples.

\section{Hazard Identification}

Colistin sulfate is an antibiotic polypeptide that has been used in cattle, pigs and poultry in Japan since 1950s. Colistin's use had been suspended clinically in humans due to the frequent adverse effects such as renal dysfunctions, and its role has been covered newly developed substitute antibiotics. In 2015, a formula of colistin methanesulfonate for injections was re-approved owing to the situation where infections with multidrug-resistant gram-negative bacilli became clinical issues in recent years.

Mechanisms of two-component regulatory systems associated with chromosomal genes have been known to operate for colistin-resistance in gram-negative bacteria. In 2015, $E$. coli strains harboring a gene ( $m c r-1)$ associated with colistinresistance on plasmids were reported in China. Up to now, colistin-resistant gene families of $m c r-1$ through 10 have been known within colistin-resistant families. The susceptibilities of E. coli and Salmonella against colistin remain high in Japan, based on the monitoring results of these bacteria isolated from the livestock from 2000 through 2017. Some strains carrying the $m c r-1, m c r-3$ and/or $m c r-5$ genes were, however, found among the isolates ${ }^{2)}$.

Both E. coli and Salmonella were potential antimicrobialresistant bacteria to be selected under the use of colistin sulfate in cattle and pigs. In cases of occurrences of human infectious diseases due to the bacteria in foods derived from livestock, these resistant bacteria could be responsible for reduction or loss of the antibiotic treatment efficacy. FSCJ thus conducted a risk assessment of E. coli and Salmonella as identified hazards.

\section{Release Assessment}

FSCJ judged the probability of selection of drug-resistant E.coli and Salmonella is low, due to the use of colistin sulfate in cattle and pigs. The following points were discussed prior to obtaining the conclusion; 1) The prevalence of $m c r$-gene was $2.0 \%$ and below in E. coli derived from domestic healthy livestock and in Salmonella derived from the sick livestock in 2015, although $m c r$-gene would be transferred among $E$. coli and between other bacteria belonging to enterobacteriaceae $^{2)}$, 2) fitness cost of bacteria with acquisition of plasmid harboring $m c r$-gene was confirmed, 3) the use of colistin was currently prohibited as a feed additive, and colistin was recognized as a second-line drug in 2018, and 4) finally, the increased rates of colistin-resistance among E. coli and Salmonella derived from livestock will be low unless otherwise the use of colistin increases.

\section{Exposure Assessment}

E. coli and Salmonella are occasionally detected in meats. However, colistin-resistant strains were scarcely isolated from these products. Considering proper cooking of the livestock products derived from cattle and pigs, the chance and extent of human exposure to the resistant bacteria were evaluated low via livestock products.

\section{Consequence Assessment}

The influence on clinical effectiveness was evaluated as moderate. Clinical importance of colistin is high as it is a recommended drug against multidrug-resistant gramnegative bacillus infections including carbapenem-resistant Enterobacteriaceae (CRE) infection caused by E. coli. Reports of multidrug-resistant gram-negative bacillus infections such as those by CRE in human cases are limited at present in Japan. Carbapenem-resistant strain has not been found among $E$. coli derived from domestic livestock. Human exposure to both colistin- and carbapenem-resistant $E$. coli from domestic animals through foods is thus unlikely at present. While E. coli or Salmonella may transfer $m c r$-gene to other bacteria, multidrug-resistant (MDR) gram-negative bacillus such as CRE acquire colistin-resistance through such $m c r$-gene transfer. As a result, other effective antibiotics may be limited. Especially, in the case of MDR Pseudomonas aeruginosa (MDRP), impact on the treatment will be significant, because colistin is often used to treat MDRP infectious disease in humans. However, transfer of $\mathrm{mcr}$-gene to MDRP was not determined in conjugal-transfer test using $m c r$-gene harboring E. coli as a donor. Therefore, the possibility that MDRP acquires colistin-resistance by transfer of $\mathrm{mcr}$ gene from livestock derived colistin resistant E.coli or Salmonella is low at present.

Antibiotics are not used generally for Salmonella-induced enteritis. The use of colistin is also not recommended for severe cases of systemic Salmonella infection under the use of other antibiotics.

\section{Risk Characterization}

Considering the hazard identification, the release assessment, the exposure assessment, and the consequence assessment, the overall estimation of the risk was low. This assessment was conducted on the premise of risk management measures taken by the MAFF in 2018. It is necessary to keep up with the latest scientific findings and information. 


\section{Acknowledgment}

FSCJ wishes to thank the members of Working Group on Antimicrobial-resistant Bacteria for preparation of the original full report.

\section{References}

1. Food Safety Commission of Japan. Antimicrobial-resistant bacteria arising from the use of colistin sulfate in the livestock (Antimicrobial-resistant Bacteria). Food Saf (Tokyo). 2017; 5(1): 24-28. PMID:6989173, doi:10.14252/ foodsafetyfscj.2016033s

2. Food Safety Commission of Japan, Tamura Y. A study on appearance and characterization of colistin-resistant bacteria (supported by research grant program No. 1703 for risk assessment on food safety from the Food Safety Commission of Japan) [in Japanese]. 2019. http://www.fsc.go.jp/fsciis/technicalResearch/show/cho99920181703. 